Journal of Nuclear Materials, Volumes 367-370, 2007, Pages 603-609

\title{
Neural Network Analysis of Charpy Transition Temperature of Irradiated Low-activation Martensitic Steels
}

\author{
G.A.Cottrell' ${ }^{1}$ R. Kemp ${ }^{2}$, H. K. D. H. Bhadeshia ${ }^{2}$, G. R. Odette ${ }^{3}$ and T. Yamamoto ${ }^{3}$
}

${ }^{1}$ EURATOM/UKAEA Fusion Association, Culham Science Centre, Abingdon, Oxon OX14 3DB, UK; ${ }^{2}$ Department of Materials Science and Metallurgy, University of Cambridge, Pembroke Street, Cambridge, CB2 3QZ, UK; ${ }^{3}$ Department of Mechanical and Environmental Engineering and Department of Materials, University of California Santa Barbara, Santa Barbara, Ca 93106, USA

\begin{abstract}
We have constructed a Bayesian neural network model that predicts the change, due to neutron irradiation, of the Charpy ductile-brittle transition temperature ( $\triangle D B T T)$ of low activation martensitic steels given a 40-dimensional set of data from the literature. The irradiation doses were $<100$ displacements per atom (dpa). Results show the high significance of irradiation temperature and $(\mathrm{dpa})^{1 / 2}$ in determining $\triangle D B T T$. Sparse data regions were identified by the size of the modelling uncertainties, indicating areas where further experimental data are needed. The method has promise for selecting and ranking experiments on future irradiation materials test facilities.
\end{abstract}

\section{Keyword codes}

E0400, F0600, H0100, L0400, M0200, N0100, R0300, S1000, T0100 
Journal of Nuclear Materials, Volumes 367-370, 2007, Pages 603-609

\section{Introduction}

Ideally, one would like to predict the behaviour of radiation-damaged materials in future fusion power plants using mechanistic models [1], allied with the experimental data from past and present facilities as well as future ones (e.g. the International Fusion Materials Irradiation Facility (IFMIF) [2]). The fusion materials problem is an example of a complex technology where the science is not fully established because of the large number of variables that determine the ultimate properties, and where ignoring any of these variables could lead to a loss of vital information [3]. The fracture toughness of irradiated steel is currently impossible to predict given a detailed description of the chemical composition, heat treatment, neutron irradiation parameters, irradiation temperature etc. Commonly used methods for empirical data fitting are limited by the need to specify a priori fitting functions and are therefore incapable of capturing unforeseen nonlinear couplings between variables, thus limiting their validity when making predictions outside the data range of the models.

In treating data, there are two kinds of errors to consider. Noise is the familiar scatter which results when an experiment is repeated. It arises because there are variables which are not controlled. Uncertainty arises because there may exist many mathematical functions which adequately represent a set of experimental data, but which behave differently in extrapolation. This modelling uncertainty becomes large when data are sparse or badly scattered and is of prime importance when dealing with non-linear fitting because it highlights the problems of extrapolation and interpolation.

This paper describes development of a Neural Network (NN) model for the ductilebrittle transition temperature shift $(\triangle D B T T)$ in irradiated Low Activation Martensitic (LAM) steels. We have previously modelled the tensile properties of such steels using a NN [4]. Toughness is related to the ability of a material to absorb energy during fracture. The Charpy test involves the measurement of the energy required to fracture a square section notched bar. 
Journal of Nuclear Materials, Volumes 367-370, 2007, Pages 603-609

Ferritic steels have a DBTT in which the fracture mode changes from ductile at high temperatures to brittle at low temperatures, and this can be measured by conducting Charpy tests at different temperatures.

\section{The Database and Network Design}

Data on the impact properties of LAM steels were compiled from the literature [5], including leading candidate alloys such as F82H (8Cr) and Eurofer 97 (9Cr) as well as the conventional T91 (9Cr-1Mo), EM10 (9Cr-1Mo) and 2.25Cr bainitic grades. This resulted in a total of about 450 experimental sets for the $\triangle D B T T$ data, Table 1. The inputs include: chemical composition, a parameter to describe the cold-work of the steel, heat treatment data, neutron irradiation dose $\phi_{i r r}(\mathrm{dpa})$ and irradiation temperature $T_{i r r}$. Some missing data prevented us from including the following inputs: dose rate, helium content, tensile yield stress and fracture mode. We have restricted the data to sub-sized Charpy specimens with cross sections ranging from $3.3 \times 3.3 \mathrm{~mm}$ to $3 \times 4 \mathrm{~mm}$.

Details of neural networks have been given by numerous authors and relevant descriptions of applications in the materials science field appear in [3]. In summary, a typical NN consists of an input layer (in this work with $\sim 40$ nodes, corresponding to the inputs in Table 1), a "hidden" layer in which the number of nodes (typically 2-15 nodes) determines the complexity of the model, and an output layer giving $\triangle D B T T$. The dataset was randomly partitioned into equal-sized training and test sets. The network is created using the training data and its ability to generalize assessed using the test data. Unlike conventional regression, the training does not simply find a "best fit" set of network weight coefficients, but, instead, assigns a probability distribution of weights to the network. This approach has the advantage that the parameters describing the distribution give a measure of the modelling uncertainty. 
Journal of Nuclear Materials, Volumes 367-370, 2007, Pages 603-609

Excessively simple or complicated models lead to large errors in predicting the test data. The correct level of model complexity corresponds to a minimum in this error and to find this we used a Bayesian inference method [6] to penalise over-complexity. The performance was further improved by making predictions using a committee of different neural networks $[3,4]$.

\section{Results}

\subsection{Model Training and Performance}

Figure 1 shows the ability of the best 14-member committee model to predict $\triangle D B T T$. There are very few outliers, i.e., points whose $\pm 1 \sigma$ modelling uncertainties do not intersect the line of unit slope.

\subsection{Significance of Individual Inputs}

It is useful to examine the significance (Fig. 2) of each input in contributing to the output; this is estimated from the variance of the network $\sigma_{\mathrm{w}}$, and is roughly equivalent to a partial correlation coefficient [6]. The normalizing and tempering temperatures and times are found to be significant inputs in predicting $\triangle D B T T$. $\triangle D B T T$ is more strongly correlated with $T_{i r r}$ than simply with dose. This result suggests that thermal mobilization of irradiation defects plays a greater role than their absolute density, but the trend is also consistent with the possible operation of irradiation damage recovery processes mitigating the $\triangle D B T T$ shift for higher irradiation temperatures. The significance of the physically-motivated function $(\mathrm{dpa})^{1 / 2}$ is larger than for dpa alone. A function of this form is consistent with models [5] either for solute depletion in a precipitation hardening mechanism, or with an excluded volume effect for the accumulation of knock-on damage defects.

The $\mathrm{C}$ and $\mathrm{Cr}$ concentrations are perceived to be important by all committee members. The addition of $\mathrm{Cr}$ alone provides very little solid solution strengthening, but $\mathrm{C}$ and 
Journal of Nuclear Materials, Volumes 367-370, 2007, Pages 603-609

Cr together promote carbide formation; it is well known that both elements determine the sequence of carbide precipitation reactions [7]. Notice that in the case of $\mathrm{Al}, \mathrm{B}, \mathrm{Co}, \mathrm{Ta}, \mathrm{Nb}$, $\mathrm{Ni}$ and $\mathrm{P}$, there is some lack of consensus between the members of the committee, indicating less reliable conclusions with these inputs.

\subsection{Model Predictions}

There are many possible ways of using the model to make predictions. In this section, we discuss some of the more interesting of these.

\subsubsection{Effect of Irradiation Temperature}

The $\triangle D B T T$ model shows that $T_{i r r}$ is the input parameter with the highest significance. Figure 3 shows its effect on $\triangle D B T T$ where the experimental points are shown to compare well with the model prediction (lines) for T91 and HT-9 (12Cr-1Mo-VW) steels with $\phi_{i r}$ of 13 and $26 \mathrm{dpa}$. The overall trend is a decrease of $\triangle D B T T$ with $T_{i r r}$. This is expected since recovery processes operate more readily at high temperatures. This is also consistent with the fact that $\triangle D B T T$ reaches an asymptotic limit with $T_{i r}$, once the competing processes of recovery and defect creation become steady. The C concentration in HT-9 is $0.2 \% \mathrm{wt}$, twice the value of that in the T91 $(0.09 \% \mathrm{wt})$, which could possibly explain why the asymptotic limit is higher for HT-9 than T91.

\subsubsection{Effect of irradiation dose}

Figure 4 shows the effect of irradiation dose on $\triangle D B T T$ for Eurofer for three values of the $T_{i r r}$. At the lowest $T_{i r r}=250^{\circ} \mathrm{C}, \triangle D B T T$ increases linearly with dose. However, at the higher $T_{i r r}$ of $350^{\circ} \mathrm{C}$, there is evidence of a possible recovery: the slope decreases and at a higher $T_{i r r}$ of $450^{\circ} \mathrm{C}$ the $\triangle D B T T$ curve shows saturation and maximum shift of $100^{\circ} \mathrm{C}$ at $\sim 20$ dpa, with a decrease at higher doses. 
Journal of Nuclear Materials, Volumes 367-370, 2007, Pages 603-609

\subsubsection{Effect of Chromium Concentration}

The variation of $\triangle D B T T$ with $\mathrm{Cr}$ concentration is shown in Fig. 5 for three values of $T_{i r r}$. A clear minimum occurs at a concentration of $\sim 9 \mathrm{wt}-\%$. The database has been checked to see whether $\triangle D B T T$ is linked to the unirradiated $D B T T$, but no correlation was found. The result is important in that it suggests there is an optimum chromium concentration to minimise in-service embrittlement. There is evidence [8] that for the higher $\mathrm{Cr}$ concentrations ( $\sim 12$ wt- $\%$ ), hardening occurs via the formation of fine-scale Cr-rich $\alpha^{\prime}$ precipitates. The hardening will give rise to $\triangle D B T T>0$. The situation is less clear for low $\mathrm{Cr}$ concentrations.

\subsubsection{Effect of Tantalum Concentration}

Tantalum was originally thought to produce carbide precipitates and thus confer strengthening in steels. However most of the Ta remains in solution after normalizing [9], and the Ta is believed to promote austenite grain refinement, thus improving fracture toughness. Although there was no strong NN committee consensus as to the significance of Ta concentration, we have nevertheless made a prediction. Figure 6 shows the predicted $\triangle D B T T$, for three values of $T_{i r r}$. Despite large uncertainties, the trend is towards lower $\triangle D B T T$ at higher Ta concentrations at all $T_{i r r}$.

\section{Summary and Conclusions}

A neural network model has been created to estimate the $\triangle D B T T$ for a database of RAFM steels, accepting, as input, an approximately 40-dimensional vector of material and irradiation parameters. The model needs no a priori fitting function. The model not only reproduces some well-established relationships but has also revealed some trends and features. These include:

- The ability to find the significant input parameters in controlling $\triangle D B T T$. The input with the highest significance is $T_{i r r}$ and the physically-based function (dpa $)^{1 / 2}$ is also 
important. Chemical composition (e.g. $\mathrm{C}$ and $\mathrm{Cr}$ as well as other elements) and preirradiation heat treatment effects are also significant.

- The tendency for $\triangle D B T T$ to decrease and saturate with increasing $T_{i r r}>\sim 450^{\circ} \mathrm{C}$.

- Recovery process for samples with high $T_{\text {irr }}$ and high doses.

- A clear minimum in $\triangle D B T T$ with $\mathrm{Cr}$ concentration after irradiation.

- Evidence for a reduction in $\triangle D B T T$ with Ta concentration after irradiation.

The model is far from comprehensive since it is constructed from an imperfect database. We were forced to exclude some data through incompleteness, highlighting the need for authors to report experimental data as fully as possible. Nevertheless, results show the sensitivity of $\triangle D B T T$ to all the inputs.

Future work will cross-correlate the model with our previous model [4] for the tensile yield stress, and predict and compare $\triangle D B T T$ with observation. This procedure should expose any biases that are related to the use of sub-sized Charpy specimens. This knowledge is important since proposed test facilities, such as IFMIF, are constrained in their high flux irradiation volumes. We believe that the $\mathrm{NN}$ method could be of value in choosing optimal experiments in this context [10]. The strength of the NN approach is to recognize and assign large modelling uncertainties in sparsely populated regions of the data space, thus allowing proposed experiments to be ranked in terms of information content.

As new materials data are reported, it will be possible to refine the model and reduce its uncertainties. Models of this type make the very best use of published information, its noise and permit extrapolation to the conditions of future fusion power plant.

\section{Acknowledgments}

We thank Ian Cook for encouragement and stimulating discussions. This work was funded jointly by the UK Engineering and Physical Sciences Research Council and by the European Community under the contract of Association between EURATOM and UKAEA, 
Journal of Nuclear Materials, Volumes 367-370, 2007, Pages 603-609

and was also supported by the US DOE Office of Fusion Energy Science (Grant \# DE-FG0394ER54275). The views and opinions expressed herein do not necessarily reflect those of the European Commission.

\section{References}

[1] Matthews, J., Int. J. of Pressure Vessels and Piping 81 (2004) 667-671.

[2] Ehrlich, K. and Möslang, A., Nuclear Instruments And Methods In Physics Research B, 139 (1998) $72-81$.

[3] Bhadeshia, H.K.D.H., Neural networks in materials science. ISIJ International, 39(10) (1999) 966 - 979.

[4] Kemp, R., Cottrell, G. A. and Bhadeshia, H. K. D. H., Odette, G. R., Yamamoto, T. and

Kishimoto, H., Journal of Nuclear Materials, 348 (2006) 311-328. $367-370$ (2007) 1586-1589.

[5] Yamamoto, T. et al., Proc. IWSMT-7 (2005).

[6] MacKay, D., Information Theory, Inference, and Learning Algorithms. 1st ed. (2003) pub. Cambridge University Press.

[7] Bhadeshia, H.K.D.H., ISIJ International, 41 (2001) 626 - 640.

[8] Suganuma, K. and Kayano, H., J. Nucl. Mater, 118 (1983) 234 - 241.

[9] Klueh, R.L., Int. Mater. Rev. 50(5) (2005) 287.

[10] Kemp, R., Cottrell, G. A. and Bhadeshia, H. K. D. H., Journal of Nuclear Materials, $367-370$ (2007) 1586-1589. 
Journal of Nuclear Materials, Volumes 367-370, 2007, Pages 603-609

Table1. Variables and their statistics used in developing the $\triangle D B T T$ model

\section{Input}

Normalising temperature $\left({ }^{\circ} \mathrm{C}\right)$

Normalising time $(\mathrm{h})$

Tempering temperature $\left({ }^{\circ} \mathrm{C}\right)$

Tempering time (h)

Coldwork $(\%)$

$\mathrm{C}(\mathrm{wt}-\%)$

$\mathrm{Cr}(\mathrm{wt}-\%)$

$\mathrm{W}(\mathrm{wt}-\%)$

Mo (wt-\%)

Ta $($ wt- $\%)$

V (wt-\%)

Si (wt-\%)

Mn (wt-\%)

$\mathrm{N}(\mathrm{wt}-\%)$

$\mathrm{Al}(\mathrm{wt}-\%)$

B (wt-\%)

Co (wt-\%)

$\mathrm{Cu}(\mathrm{wt}-\%)$

$\mathrm{Nb}(\mathrm{wt}-\%)$

$\mathrm{Ni}(\mathrm{wt}-\%)$

P (wt-\%)

S (wt-\%)

Ti (wt-\%)

$\mathrm{Zr}(\mathrm{wt}-\%)$

Irradiation temperature $\left({ }^{\circ} \mathrm{C}\right)$
Data Range

$0-1100$

$0-2$

$0-780$

$0-2.5$

$0-27$

$0.005-0.2$

$0-12$

$0-2.12$

$0-1$

$0-0.48$

$0-0.314$

$0-0.4$

$0.04-1.35$

$0.0007-0.06$

$0.001-0.054$

$0-0.009$

$0.0024-0.02$

$0.0017-0.035$

$0.0001-0.2$

0.005 - 2

$0.002-0.016$

$0.0002-0.008$

0.001

$0-0.059$

$60-550$
Mean \pm s.d.

$1024.6 \pm 95.7$

$0.67 \pm 0.33$

$740.2 \pm 83.0$

$1.71 \pm 0.502$

$0.941 \pm 4.958$

$0.115 \pm 0.025$

$8.85 \pm 1.951$

$1.009 \pm 0.889$

$0.207 \pm 0.340$

$0.033 \pm 0.046$

$0.218 \pm 0.064$

$0.173 \pm 0.123$

$0.496 \pm 0.269$

$0.018 \pm 0.012$

$0.015 \pm 0.015$

$0.003 \pm 0.003$

$0.006 \pm 0.004$

$0.012 \pm 0.011$

$0.035 \pm 0.065$

$0.181 \pm 0.332$

$0.007 \pm 0.005$

$0.004 \pm 0.002$

$0.006 \pm 0.003$

$0.007 \pm 0.017$

$352.7 \pm 71.041$ 
Journal of Nuclear Materials, Volumes 367-370, 2007, Pages 603-609

Irradiation dose (dpa)

$0-100$

$5.42 \pm 10.21$

Range of $\triangle D B T T\left({ }^{\circ} \mathrm{C}\right)$

$(-) 39-335$

$67.21 \pm 63.66$ 
Journal of Nuclear Materials, Volumes 367-370, 2007, Pages 603-609

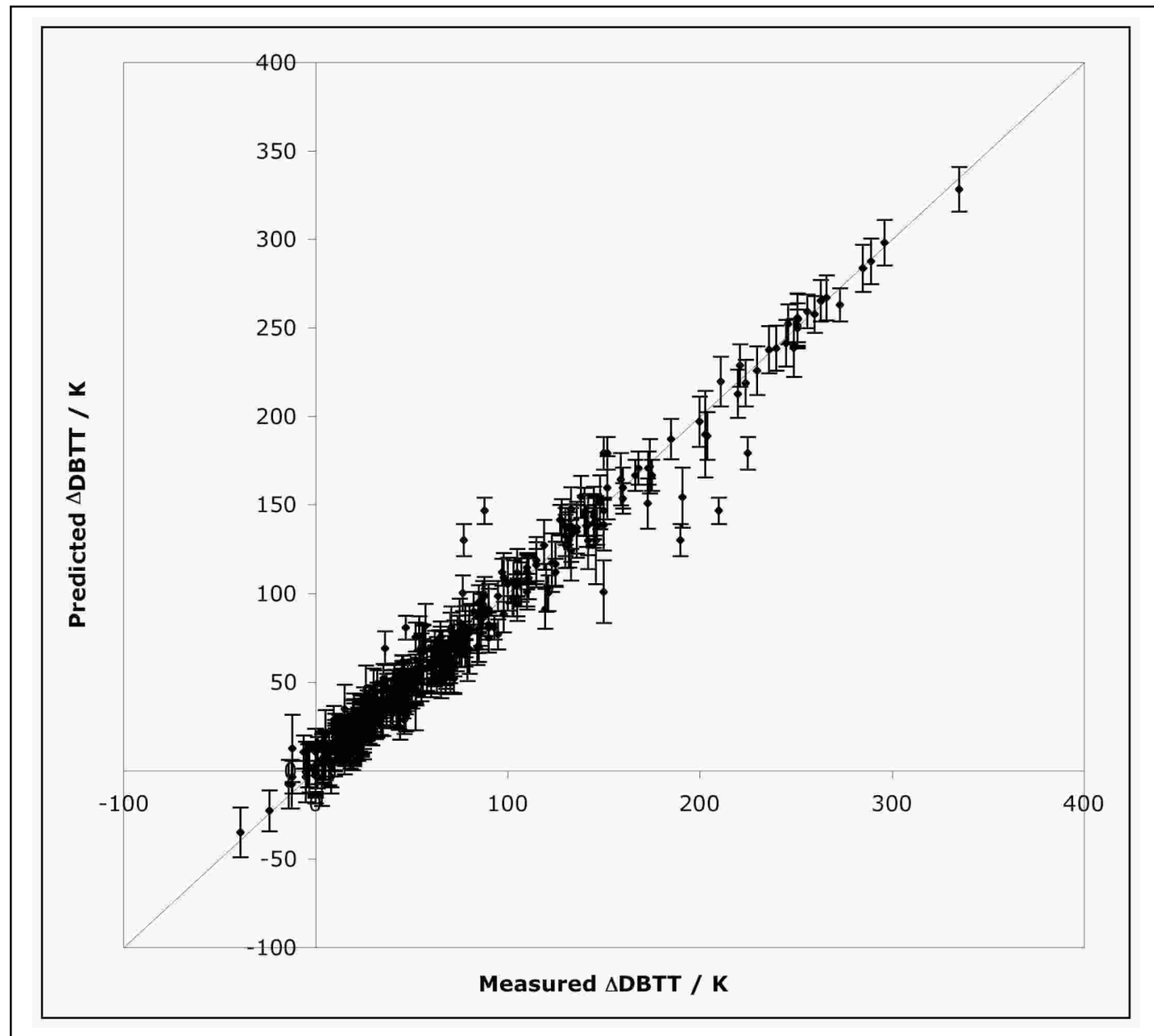

Fig 1. Model-predicted change in $D B T T$ ( $\triangle D B T T$ after irradiation) versus the measured value. 


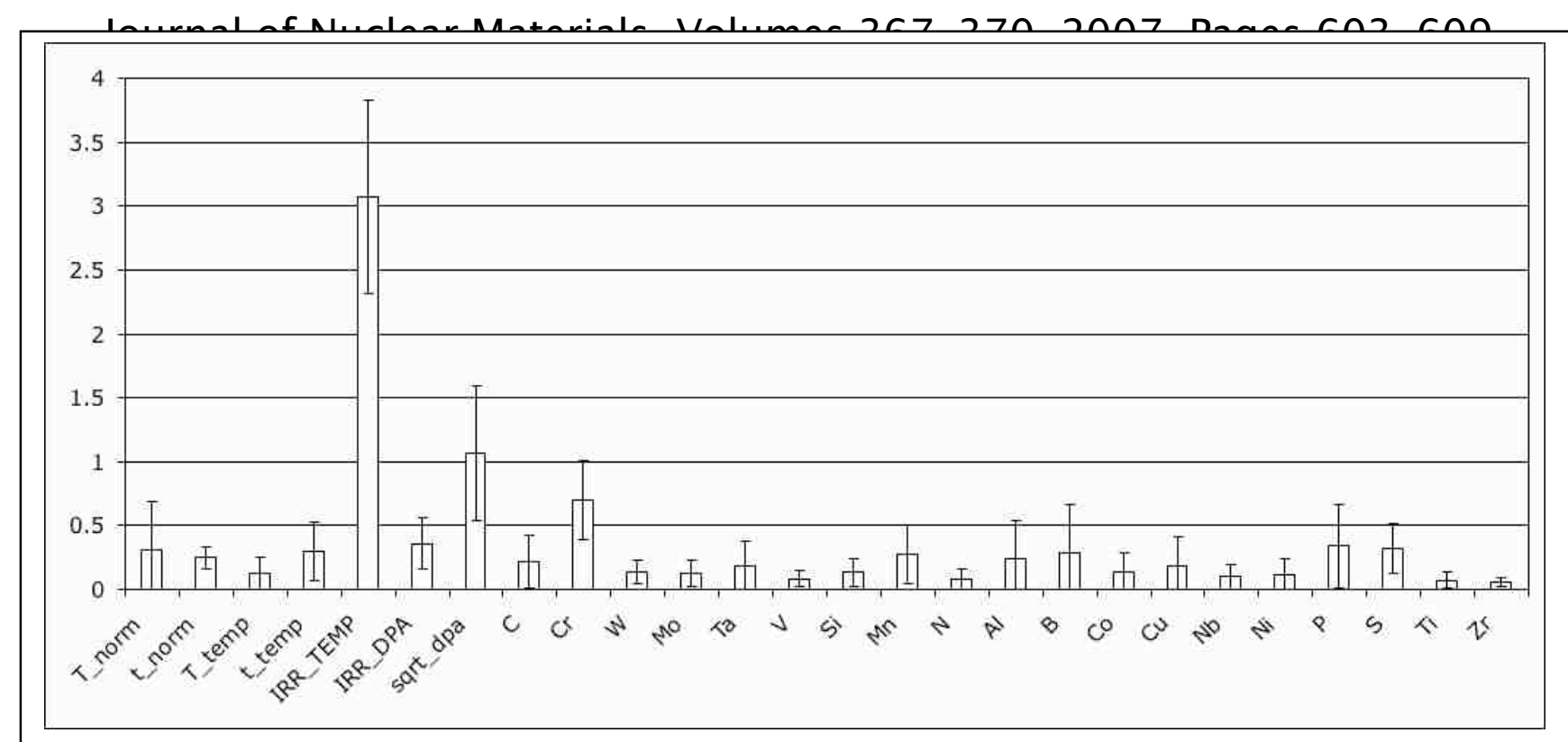

Fig. 2. Perceived model significances for $\triangle D B T T$ of heat treatment parameters, irradiation parameters and chemical element composition. The histograms represent the mean significance of all 14 committee members for each parameter and the error bars the standard deviation within each group.

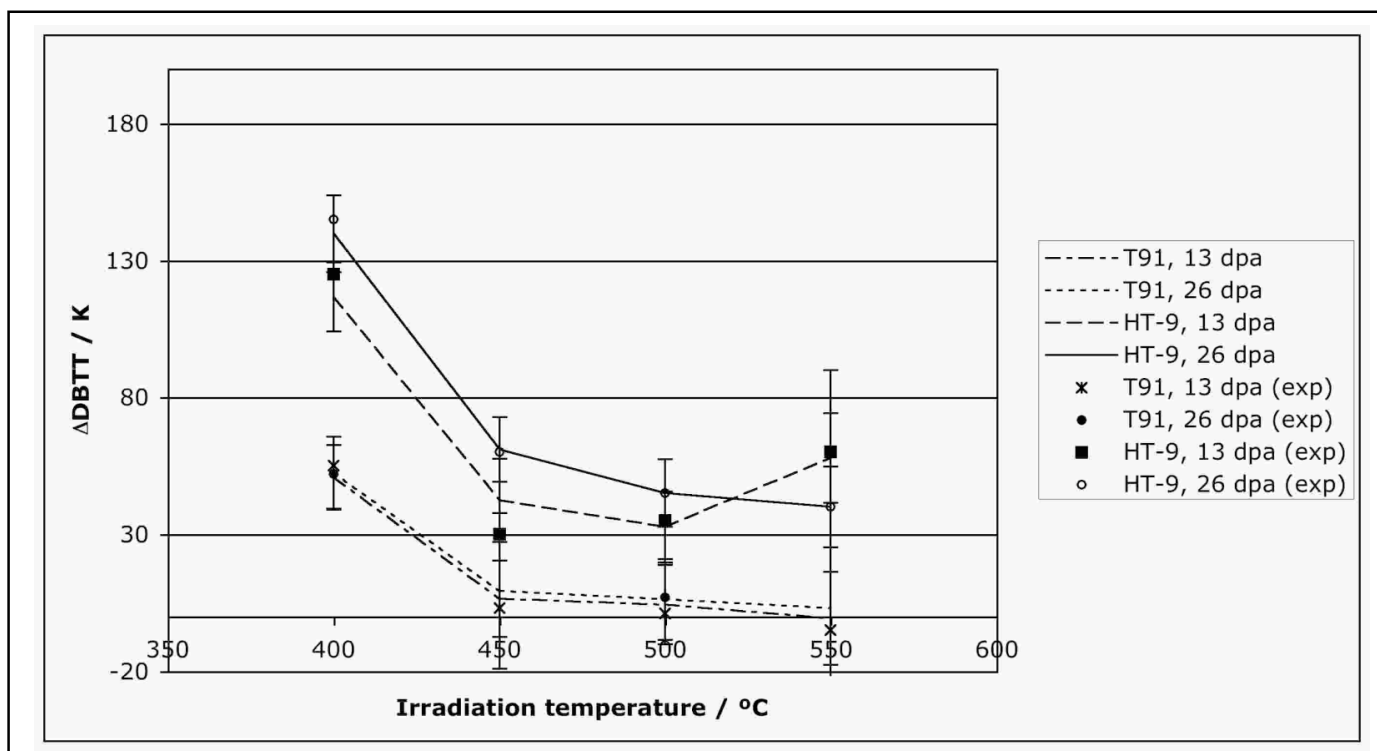

Fig 3. Variation of $\triangle D B T T$ with irradiation temperature, comparing model predictions (lines) with the data (points) for T91 and HT-9 steels at 13 and 26 dpa. 
Journal of Nuclear Materials, Volumes 367-370, 2007, Pages 603-609

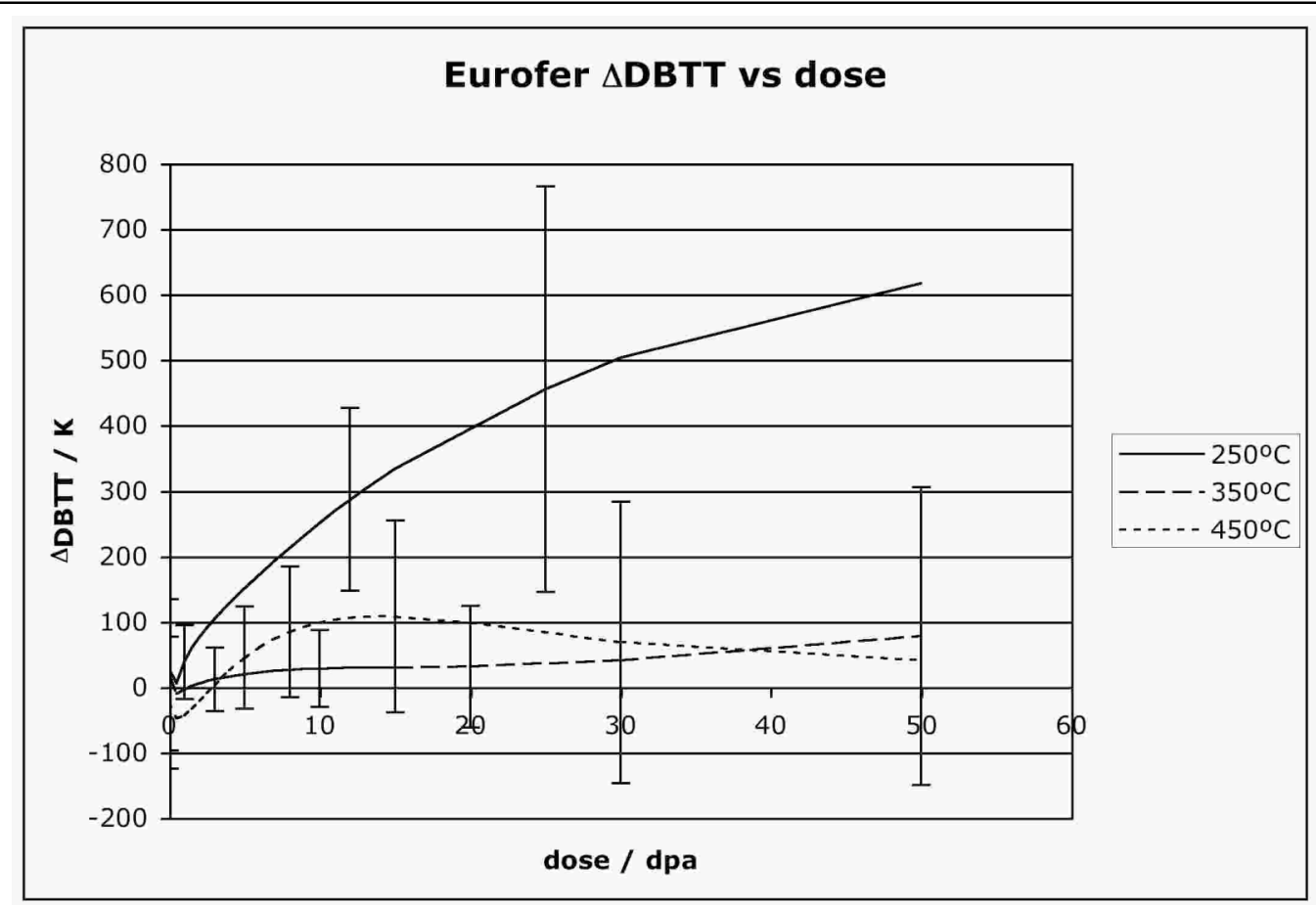

Fig 4. Model prediction: variation (with modelling uncertainties) of $\triangle D B T T$ with the irradiation dose for Eurofer for three irradiation temperatures.

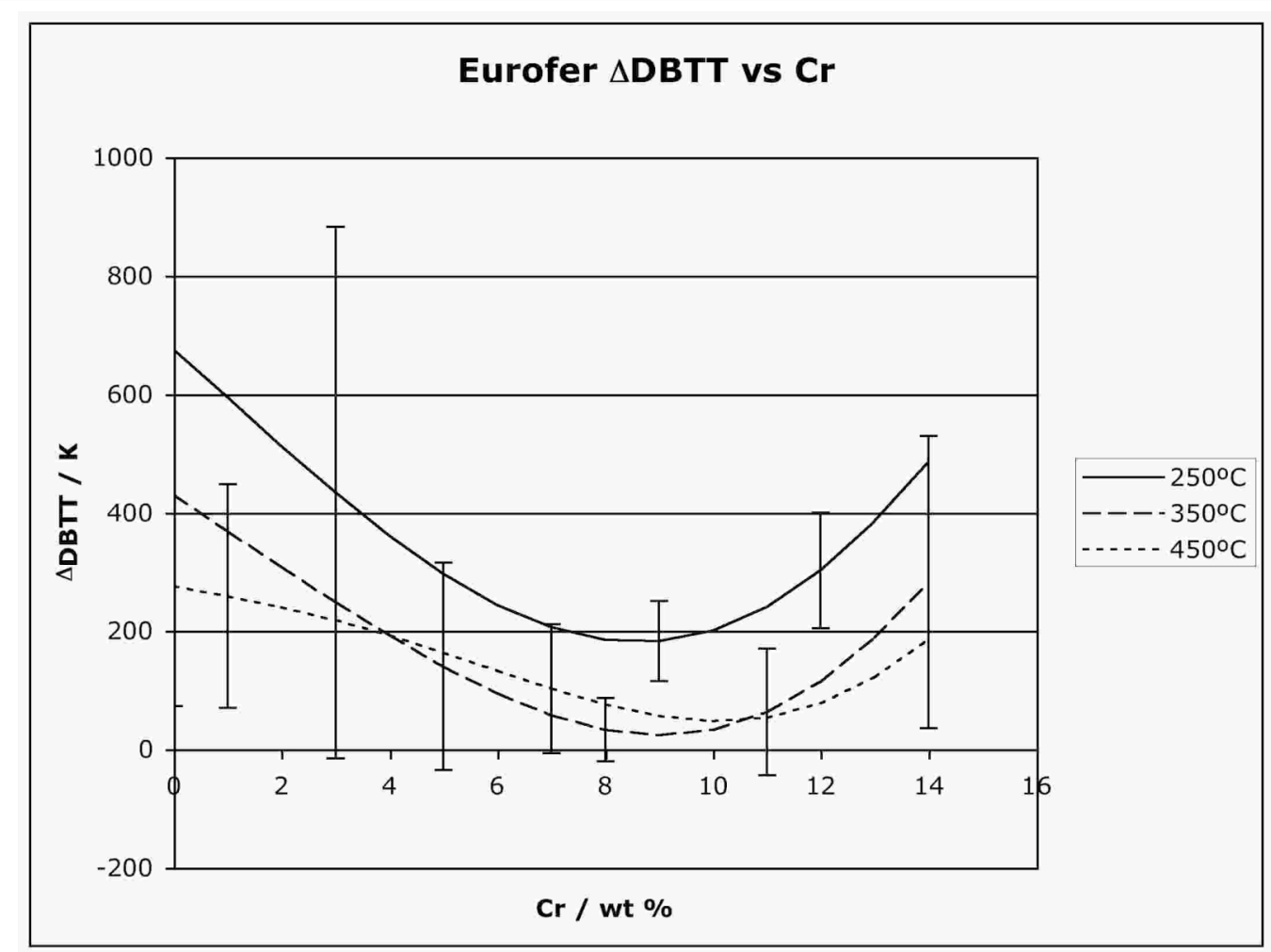

Fig 5. Model prediction: variation (with modelling uncertainties) of $\triangle D B T T$ with the $\mathrm{Cr}$ concentration for Eurofer for three irradiation temperatures and a dose of $10 \mathrm{dpa}$. 
Journal of Nuclear Materials, Volumes 367-370, 2007, Pages 603-609

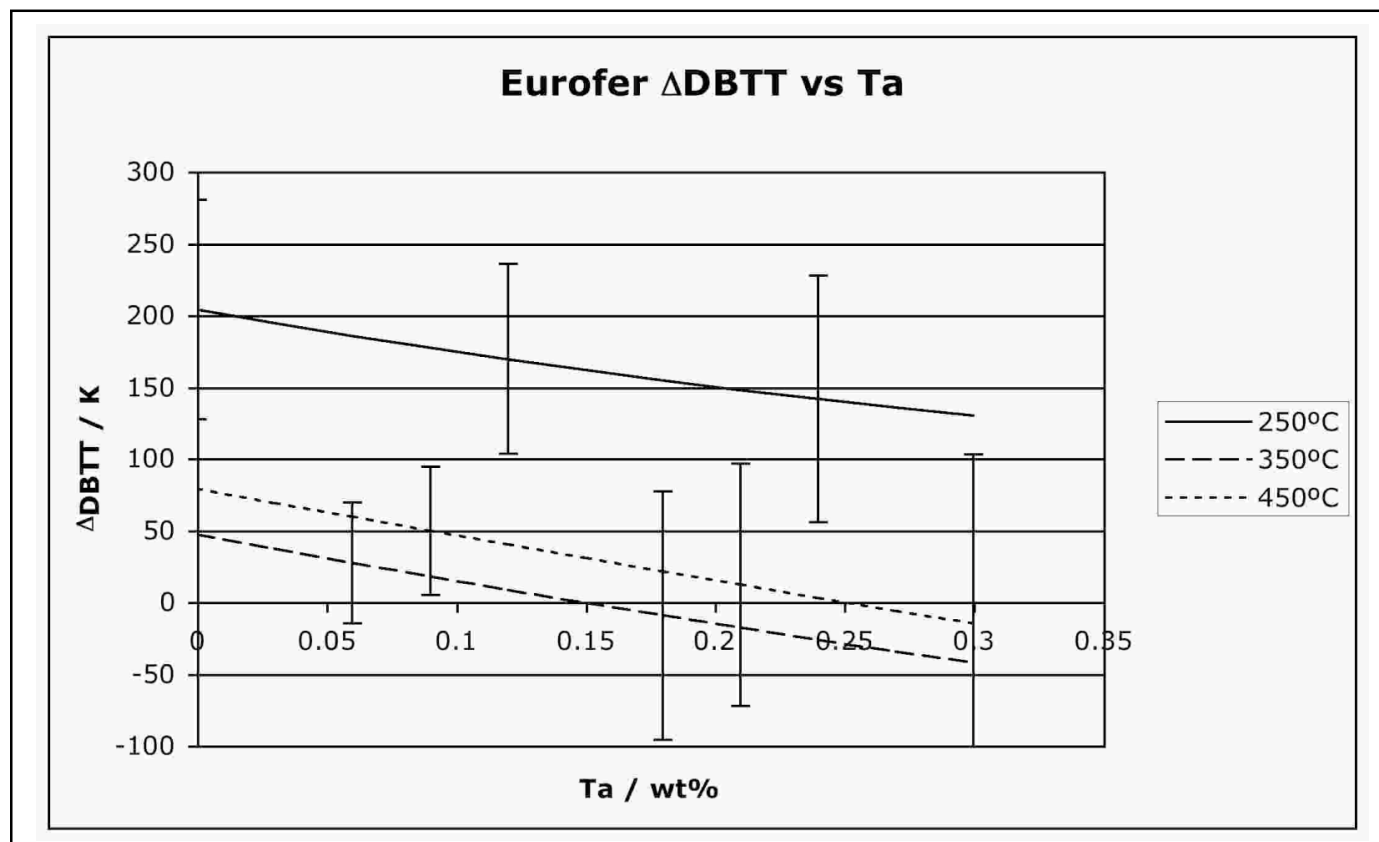

Fig 6. Model prediction: variation (with modelling uncertainties), of $\triangle D B T T$ on the Tantalum concentration for Eurofer for three irradiation temperatures and a dose of $10 \mathrm{dpa}$. 